\title{
Efeito da Consequência Programada Sobre a Estabilidade da Taxa de Respostas em Esquema FI ${ }^{1}$
}

\author{
Luiz Alexandre Barbosa de Freitas ${ }^{a^{*}}$, Raquel Fernanda Ferreira Lacerda ${ }^{b} \&$ \\ Carlos Eduardo Costa ${ }^{b}$ \\ ${ }^{\mathrm{a}}$ Universidade Federal do Mato Grosso, Cuiabá, Brasil; ${ }^{\mathrm{b}}$ Universidade Estadual de Londrina, Londrina, Brasil
}

\begin{abstract}
RESUMO
Estudos sugerem que a taxa de respostas de humanos em FI pode ser afetada pelo tipo de consequência programada. O objetivo foi verificar se diferentes consequências programadas para humanos respondendo em FI influenciariam o tempo necessário para atingir um critério de estabilidade quantitativo e se a estabilidade se manteria após ter sido atingida. Quinze universitários foram expostos a um FI $30 \mathrm{~s}$ por 10 horas (sessões de $30 \mathrm{~min}$ ) em duas condições com consequências distintas para o responder (pontos vs. pontos trocados por dinheiro). Os resultados sugerem que a consequência programada não é suficiente para influenciar o tempo necessário para atingir o critério de estabilidade, embora pareça afetar a manutenção da estabilidade.
\end{abstract}

Palavras-chave: esquemas de reforçamento; intervalo fixo; critérios de estabilidade; tipo de reforçador; humanos.

\section{ABSTRACT \\ Effects of the Scheduled Consequence on Stability of Response Rate in FI}

Studies suggest that the response rate of humans under FI may be affected by the type of reinforcer. The purpose of this study was to determine whether the type of scheduled consequence for humans under FI would affect the time required to reach a quantitative stability criterion and whether stability would be maintained after being reached. Fifteen college students were exposed to a FI $30 \mathrm{~s}$ for 10 hours (30 min sessions) under two conditions with different consequences for responses (points vs. money). The results suggest that the type of reinforcer is not sufficient to affect the time required to reach the stability criterion, although its maintenance seems to be influenced by this variable.

Keywords: schedules of reinforcement; fixed interval; stability criteria; type of reinforcer; humans.

O desempenho estável em delineamentos de casoúnico serve de parâmetro para decisões experimentais (e.g., o momento de inserir a variável experimental) e como predição do desempenho futuro. Nesses delineamentos, o controle estatístico é substituído pelo controle experimental. $\mathrm{O}$ desempenho estável indicaria que o comportamento observado não estaria sendo afetado por variáveis estranhas ao procedimento $(\mathrm{Ba}-$ ron \& Perone, 1998; Kazdin, 1982; Matos, 1990; Perone, 1991; Sidman, 1960).

Um modo de aferir a estabilidade do comportamento é expor os organismos à condição experimental por um período fixo de tempo, como feito, por exemplo, por Weiner $(1964,1969)$. A confiabilidade nesse tipo de critério pode ser baixa, pois organismos que ainda não estejam com o comportamento estável podem ser incluídos no grupo daqueles com o comportamento estabilizado - exceto se o pesquisador tiver ampla experiência na área e, com base em dados anteriores, souber a duração mínima para que os sujeitos mais lentos apresentem desempenho estável (Baron \& Perone, 1998; Sidman, 1960). No entanto, esse critério não impede que a estabilidade seja verificada posteriormente utilizando métodos diferentes. Outro modo é a inspeção visual da taxa de respostas (e.g., Buskist, Bennett \& Miller, 1981; Buskist, Miller \& Bennett, 1980), no qual se verifica a faixa em que o comportamento varia, bem como a ocorrência ou não de

Endereço para correspondência: Luiz Alexandre Barbosa de Freitas - luizalexandrefreitas@gmail.com 
ciclos e tendências. Esse critério é mais tolerante a tendências e ciclos que o critério quantitativo (Baron \& Perone, 1998; Johnston \& Pennypacker, 1993).

Uma terceira maneira de avaliar a estabilidade é pelo critério quantitativo. A variação na taxa de respostas pode ser avaliada em termos absolutos ou relativos. Nos critérios quantitativos absolutos, a variação é determinada em unidades fixas. Por exemplo, um comportamento que ocorra com taxa baixa (e.g., cinco respostas por minuto) será considerado estável se tiver uma variação de até duas respostas por minuto, para mais ou para menos. Nos critérios quantitativos relativos, a variação considerada aceitável é determinada em termos percentuais (Perone, 1991). Um critério de estabilidade quantitativo relativo é o de Schoenfeld, Cumming e Hearst (1956), retomado por Cumming e Schoenfeld (1960). Nesses estudos, o critério considerou as taxas de respostas das seis sessões mais recentes. Calcularam-se as médias do primeiro bloco de três sessões e do segundo bloco de três sessões (cálculo de duas submédias), e a média do bloco inteiro de seis sessões (média geral). O comportamento era julgado estável se a diferença entre as submédias, dividida pela média geral, fosse menor que 5\% (ver fórmula em Costa \& Cançado, 2012). Muitos estudos com humanos e não-humanos utilizaram esse critério (e.g., Freeman \& Lattal, 1992, Experimento 3; Galizio, 1979; Lattal, 1970; Okouchi, 2002). Em geral, eles diferem quanto ao número de sessões por bloco e quanto à porcentagem máxima de variação permitida. Galizio, por exemplo, em um experimento com humanos, mediu a estabilidade em blocos de quatro sessões e a variação máxima permitida foi de $15 \%$.

Uma característica dos critérios de estabilidade quantitativos é quanto ao rigor para tolerar variação no comportamento. $\mathrm{O}$ rigor de um critério de estabilidade depende da taxa geral de respostas (Baron \& Perone, 1998; Perone, 1991; Sidman, 1960). No caso de um critério relativo, como o de Schoenfeld et al. (1956), ele será tão mais rigoroso quanto menor for a taxa de respostas. Por exemplo, se um critério de estabilidade relativo com variação máxima de $15 \%$ fosse utilizado para avaliar a variação da taxa de respostas em um esquema de reforço que produzisse entre 2 e 4 respostas por minuto $(\mathrm{R} / \mathrm{min})$, dificilmente ele seria atingido, porque uma variação de $1 \mathrm{R} / \mathrm{min}$ representaria de 25 a $50 \%$ de variação. Seria melhor utilizar um critério absoluto que determinasse que a variação aceitável fosse, por exemplo, de 1 ou $2 \mathrm{R} / \mathrm{min}$. Por outro lado, aplicar um critério absoluto para comportamentos em taxas altas também seria inadequado, pois quando a taxa de respostas é muito alta, a variação (em número absoluto de respostas) tende a ser mais alta do que quando as taxas são baixas e, portanto, o mais indicado seria um critério relativo (Perone, 1991).

Apesar dessa característica, o critério de estabilidade relativo tem sido empregado mesmo em procedimentos que podem produzir taxas de respostas baixas. Por exemplo, Okouchi (2002), em um estudo com humanos em FI, adotou o critério de estabilidade utilizado por Schoenfeld et al. (1956). Nesse estudo, 32 universitários foram expostos a um FI $60 \mathrm{~s}$. Para 28 participantes a exposição durou oito sessões (de cerca de 20 minutos cada uma) ou até que o critério de estabilidade fosse atingido. Para os outros quatro participantes, a duração foi de 60 sessões no total, incluindo aquelas em que o critério de estabilidade ainda não havia sido atingido para cada participante. Dos 32 participantes, somente dois não atingiram o critério de estabilidade em oito sessões. A taxa de respostas, aparentemente, teve alguma influência no número de sessões necessário para atingir o critério de estabilidade. Embora alguns participantes com taxas de respostas baixas tenham atingido o critério de estabilidade, os dois únicos participantes que não o atingiram também responderam em taxas relativamente baixas (menos de $30 \mathrm{R} / \mathrm{min}$ ). Uma análise de correlação de Pearson (não oferecida pelo autor) entre o número de sessões necessário para que o critério fosse atingido e a taxa de respostas (dados de Okouchi, Tabela 1, p. 177) indicou que os participantes com taxas mais altas, em geral, atingiram o critério de estabilidade com um número menor de sessões $(r=-0,5169, p=0,0034)$.

Outro resultado interessante do estudo de Okouchi (2002) foi a diversidade de padrões de respostas observados no momento em que o critério de estabilidade foi atingido. Os padrões foram agrupados em: taxa de respostas alta e constante ao longo de cada intervalo entre reforços (IRI), com pouca ou nenhuma pausa pós-reforço (PRP); taxa de respostas moderada nos IRIs, com PRP curta; padrão de break-and-run ${ }^{2}$ e uma ou duas respostas no final do IRI, com PRP longa. Em relação aos quatro participantes com exposição prolongada ao FI, dois responderam em taxas altas e constantes (quase sempre acima de $300 \mathrm{R} / \mathrm{min}$ ) sem PRP, um emitiu taxas de respostas baixas (menos de $31 \mathrm{R} / \mathrm{min}$ ) com PRP longa e um respondeu em taxas moderadas (entre 50 e $150 \mathrm{R} / \mathrm{min}$, aproximadamente) com períodos de taxas relativamente baixas (abaixo de $50 \mathrm{R} / \mathrm{min}$ ). Além disso, para esses quatro participantes, uma vez atingido o critério de estabilidade, as 
taxas de respostas mantiveram-se relativamente estáveis até as sessões finais.

Essa variabilidade entre participantes, encontrada por Okouchi (2002), descreve o que é comumente observado nos experimentos com humanos expostos ao FI (e.g., Bentall, Lowe \& Beasty, 1985; Buskist et al., 1980; Costa, Banaco \& Becker, 2005; Matthews, Shimoff, Catania \& Sagvolden, 1977; Weiner, 1969, Experimento 1). O padrão encontrado com não-humanos é de pausa pós-reforço, seguida de um aumento gradual (scallop) ou abrupto (break-and-run) na taxa de respostas até a liberação do próximo reforço (Cumming \& Schoenfeld, 1958; Ferster \& Skinner, 1957).

A diversidade de padrões de desempenhos e taxas de respostas produzidas com humanos em FI pode estar relacionada às diferentes consequências programadas durante o experimento. Em um estudo que manipulou essa variável, Costa et al. (2005) expuseram 11 universitários a um FI $10 \mathrm{~s}$, distribuídos em três condições de reforço: para três, a consequência por cumprir a contingência era pontos trocados por fotocópias (Condição 1-Fotocópias); para três, era pontos trocados por dinheiro (Condição 2-Dinheiro) e para cinco, era apenas a produção dos pontos (Condição 3-Pontos). Os três participantes da Condição 2-Dinheiro emitiram taxas de respostas altas e constantes nas três sessões experimentais de 15 min cada. Nas outras condições, embora taxas altas e constantes também tenham sido observadas, alguns participantes emitiram baixas taxas de respostas e alguns emitiram altas taxas de respostas no início do FI, as quais diminuíram com a exposição continuada ao FI. Pontos trocados por dinheiro pareceram favorecer taxas de respostas altas e constantes em FI, embora o evento consequente empregado não seja a única variável responsável por esse desempenho, uma vez que taxas altas e constantes também foram observadas no desempenho de alguns participantes das outras condições. Esses resultados corroboram os encontrados em outros estudos que indicaram que o desempenho pode variar em função da consequência utilizada (e.g., Costa et al., 2008; Mace, Mauro, Boyajian \& Eckert, 1997; Salgado, Cançado \& Costa, 2011; Ward, 1976; Weiner, 1972).

Se a taxa de respostas pode afetar tanto o rigor do critério de estabilidade (Baron \& Perone, 1998; Perone, 1991), quanto o número de sessões necessário para que um determinado critério de estabilidade seja atingido (cf. Okouchi, 2002) e se a consequência progra- mada pode afetar as taxas de respostas de humanos em alguns esquemas de reforço (FI - Costa et al., 2005; FR - Weiner, 1972), então, o critério de estabilidade da taxa de respostas em FI poderia ser atingido mais ou menos rapidamente dependendo do tipo de consequência programada? Os resultados de Okouchi (2002), como apontado anteriormente, sugerem que, quando as taxas de respostas foram altas, o número de sessões requerido para que o critério de estabilidade fosse atingido foi menor do que quando as taxas foram baixas. Entretanto, no estudo de Okouchi, todos os participantes foram pagos pelo desempenho na tarefa experimental e para cada hora de participação no experimento. Além disso, o estudo de Costa et al. sugeriu que pontos trocados por dinheiro tenderam a gerar taxas de respostas mais altas do que quando os eventos consequentes eram pontos apenas, quando os participantes foram expostos por 45 minutos ao FI com diferentes consequências programadas (três sessões de 15 minutos). Essa relação entre a consequência programada e a taxa de respostas seria mantida mesmo com uma exposição maior (e.g., 10 horas) ao FI?

O objetivo do presente estudo foi (a) verificar se o tipo de consequência programada (pontos trocados por dinheiro ou pontos apenas) para o comportamento de humanos em um FI teria efeito sobre o número de sessões necessário para que um critério de estabilidade quantitativo relativo da taxa de respostas fosse atingido; (b) verificar se a estabilidade, definida por esse critério, se manteria após ter sido atingida (i.e., se a taxa de respostas das sessões em que a estabilidade foi atingida seria preditiva do desempenho futuro - cf. Kazdin, 1982); e (c) descrever o comportamento de humanos em FI, em uma exposição "prolongada" (10 h), em função da consequência programada (pontos trocados por dinheiro ou pontos apenas).

\section{MÉTODO}

\section{Participantes}

Participaram 15 universitários, de ambos os sexos, de 17 a 25 anos de idade, de diferentes cursos (inclusive alunos do $1^{\circ}$ ano de Psicologia), sendo 11 de uma universidade pública (identificados pela letra U) e quatro de uma faculdade privada (identificados pela letra P). Nenhum tinha experiência prévia em experimentos com condicionamento operante, nem haviam tido aulas sobre esquemas de reforço. 


\section{Ambientes}

No laboratório da universidade pública, havia duas salas de aproximadamente $3 \mathrm{~m}^{2}$ cada. Cada uma continha uma mesa, uma cadeira, um computador com teclado e mouse e um gravador com fone de ouvido que emitia um ruído branco para isolamento acústico. No outro laboratório, da faculdade particular, havia duas salas de aproximadamente $6 \mathrm{~m}^{2}$ cada. Em cada sala havia uma mesa, uma cadeira e um computador com teclado e mouse. Essas salas possuíam bom isolamento acústico, não sendo necessária a utilização de ruído branco.

\section{Equipamentos e Instrumentos}

Foram utilizados quatro computadores do tipo PC, com monitor em cores de 14 polegadas, mouse e teclado padrão. Para a coleta de dados foi utilizado o software ProgRef v3.1 (Costa \& Banaco, 2002). A primeira tela do software consistia em um fundo cinza, contendo um botão com a palavra "Iniciar" na posição central superior da tela. Ao clicar sobre esse botão, a sessão experimental era iniciada com a apresentação de uma segunda tela que consistia em um fundo cinza, contendo um retângulo no centro inferior da tela (botão de respostas), um contador de pontos no centro superior da tela e um retângulo no canto superior direito da tela (botão de resposta de consumação). Para o cálculo da estabilidade da taxa de respostas foi utilizado o software Stability Check (Costa \& Cançado, 2012).

\section{Procedimento}

Antes da primeira sessão, o participante lia e assinava um Termo de Consentimento Livre e Esclarecido $\left(\mathrm{TCLE}^{3}\right)$. Em linhas gerais, o participante era informado no TCLE que o objetivo da pesquisa era "estudar algumas variáveis que possam afetar o modo como as pessoas se comportam em determinadas situações"; que deveria ganhar o maior número de pontos usando apenas o mouse; o número e a duração das sessões; que deveria usar um fone de ouvido durante as sessões (quando esse era o caso) e que haveria um sorteio na presença do participante para definir o grupo do qual faria parte. O TCLE também especificava para todos os participantes que um grupo receberia pontos trocados por dinheiro ao final de cada sessão (Grupo 1-Dinheiro) e o outro grupo receberia pontos apenas (Grupo 2-Pontos) e que eles permaneceriam no grupo para o qual foram sorteados até a última sessão do estudo. Os participantes do Grupo 1-Dinheiro re- cebiam, ao final de cada sessão, $\mathrm{R} \$ 0,05$ por ponto ganho. Após a assinatura do TCLE era solicitado ao participante que lesse uma folha com as seguintes instruções:

Esse trabalho não se trata de uma pesquisa sobre inteligência ou personalidade. Seu objetivo será ganhar pontos utilizando apenas o mouse. Os pontos aparecerão em uma janela (contador) que se localizará na parte superior da tela do computador na posição central. $O$ experimentador não está autorizado a dar qualquer informação adicional. Caso haja dúvidas, releia o texto e prossiga o experimento. Bom trabalho!

Antes do início de cada sessão, era solicitado ao participante que deixasse todo seu material, incluindo relógio e aparelho celular em uma mesa fora do local utilizado para a coleta de dados. Em seguida, aos participantes da universidade pública, era pedido que colocassem o fone de ouvido e não o retirassem até o final da sessão. A sessão tinha início assim que o participante clicasse o botão do mouse sobre um botão escrito "Iniciar".

Todos os participantes, independentemente do grupo e da instituição à qual faziam parte, foram submetidos a um FI 30 s. Os participantes deveriam responder pressionando o botão esquerdo do mouse com o cursor sobre o botão de respostas. Um smile aparecia no canto superior direito da tela assim que a primeira pressão no botão de resposta ocorresse após a passagem de 30 segundos desde o aparecimento do último smile ou do início da sessão. O participante deveria, então, clicar com o cursor sobre o botão de resposta de consumação para que o smile desaparecesse e um ponto fosse creditado no contador de pontos. O intervalo FI tinha início logo após o aparecimento do smile e não após a resposta de consumação. Portanto, o tempo necessário para deslocar o cursor do mouse do botão de respostas até o botão de resposta de consumação e clicar sobre ele era computado como parte da pausa pós-reforço. Essa programação da resposta de consumação é semelhante à utilizada na maioria das pesquisas com ratos em que água é usada como evento consequente (i.e., uma dada pressão à barra aciona o bebedouro, um novo intervalo é iniciado e o tempo que o rato passa lambendo o bebedouro está dentro do intervalo do FI).

Se a contingência fosse cumprida no botão de respostas mais de uma vez sem que o participante clicasse no botão de resposta de consumação, outro smile não aparecia (somente o que já estava na tela desde o 
momento em que a contingência foi cumprida permanecia na tela) e o participante deixaria de ganhar mais pontos enquanto não emitisse a resposta de consumação. Enquanto um smile estivesse visível na tela do computador o software continuava reiniciando o intervalo do FI para a primeira resposta que ocorresse após 30 segundos.

Foram realizadas 20 sessões com a duração de 30 minutos cada, totalizando 10 horas com cada participante. As sessões experimentais ocorreram diariamente (exceto sábados, domingos e feriados). Eram realizadas, no máximo, duas sessões por dia, com intervalo mínimo de cinco minutos entre elas.

A estabilidade da taxa de respostas foi avaliada em blocos de quatro sessões (i.e., Sessões 1 a 4; 2 a 5; 3 a 6; 4 a 7 e assim por diante até o bloco com as Sessões 17 a 20, cf., Costa \& Cançado, 2012), segundo o critério de Schoenfeld et al. (1956). A taxa era considerada estável se o índice de estabilidade (IE) fosse menor ou igual a $15 \%$ (assim como em Galizio, 1979). Foi realizada, também, a análise de correlação de Pearson entre o número de sessões necessárias para atingir o critério de estabilidade e (a) a taxa de respostas; (b) o número de pontos obtidos.

\section{RESULTADOS}

As Figuras 1 e 2 apresentam a taxa de respostas (círculos cheios e eixo y à esquerda) e o número de pontos obtidos (círculos vazios e eixo y à direita) de cada participante dos Grupos 1 e 2, respectivamente, ao longo das 20 sessões de FI. Os blocos de quatro sessões, nos quais o critério de estabilidade foi atingido, estão indicados pelos retângulos cinza. Os valores abaixo de cada gráfico se referem às sessões (entre parênteses) nas quais o critério de estabilidade (IE $\leq$ $15 \%$ ) foi atingido e aos valores percentuais de variação obtidos nesses blocos de sessões. Como não foram observadas diferenças sistemáticas no desempenho dos participantes (taxa de respostas, número de sessões para atingir o critério de estabilidade, número de pontos obtidos etc.) da universidade pública e da faculdade privada, as descrições e análises dos resultados, de forma geral, não foram pautadas nessa diferença.

Observa-se nas Figuras 1 e 2 que, para a maioria dos participantes, o critério de estabilidade foi atingido, aproximadamente, no primeiro terço da exposição às sessões de FI. Seis de oito participantes do Grupo 1-Dinheiro (U1, U2, U3, U4, P1 e P2) e cinco de sete participantes do Grupo 2-Pontos (U7, U9, U10, U11 e P3) atingiram o critério de estabilidade até a $7^{\mathrm{a}}$ sessão. Dos demais participantes de ambos os grupos, três atingiram o critério de estabilidade até a $12^{\mathrm{a}}$ sessão (U5 do Grupo 1-Dinheiro, U8 e P4 do Grupo 2Pontos) e um atingiu o critério após 17 sessões (U6 do Grupo 1-Dinheiro).

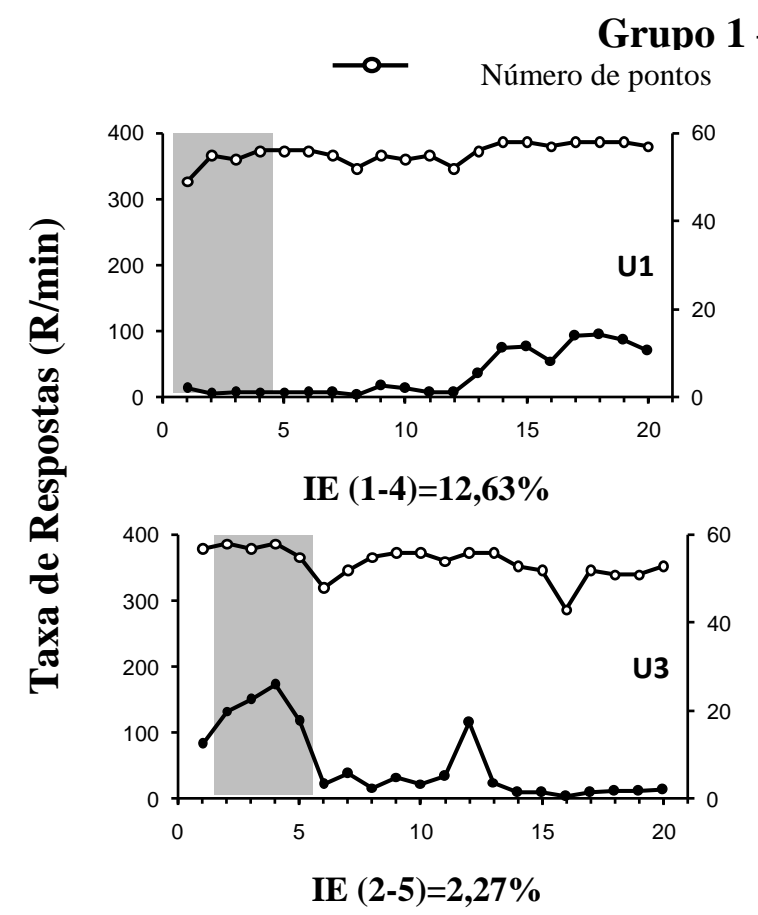




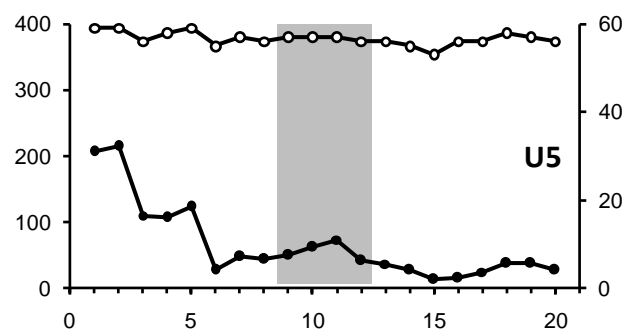

IE $(9-12)=2,88 \%$

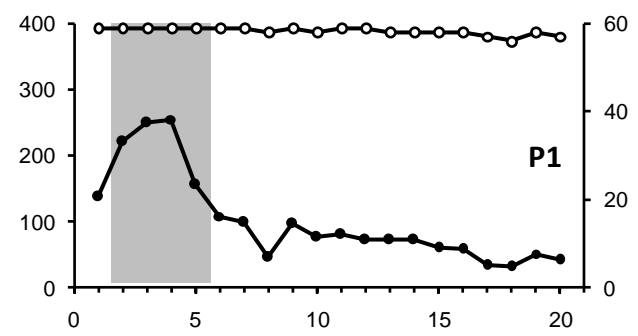

IE $(2-5)=13,61 \%$

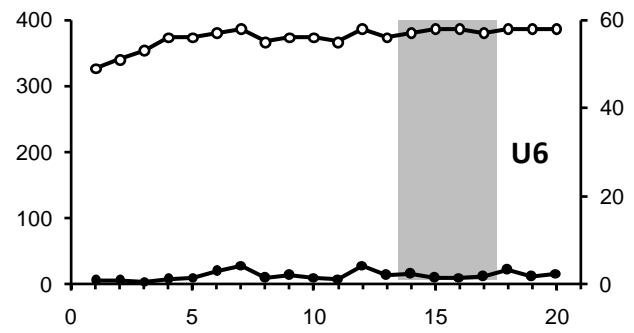

IE (14-17)=13,02\%

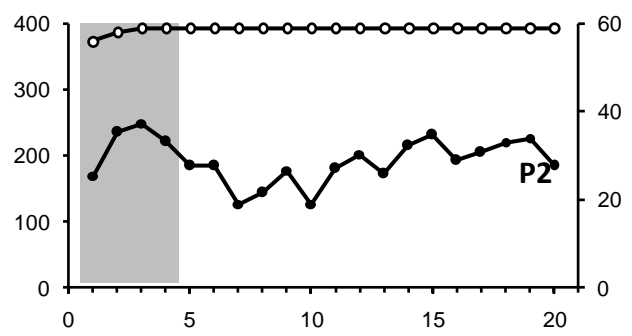

IE $(1-4)=14,9 \%$

\section{Sessões}

Figura 1. Taxa de respostas em cada sessão para os participantes do Grupo 1 - Dinheiro (representada pela linha com círculos preenchidos e valores no eixo y à esquerda). O número de pontos obtidos em cada sessão está representado pela linha com círculos vazios e valores no eixo y à direita. As sessões dentro dos retângulos verticais representam o bloco de quatro sessões em que o critério de estabilidade foi atingido. O Índice de Estabilidade (IE) e o bloco de sessões em que foram alcançados (nos parênteses) estão descritos abaixo de cada gráfico.

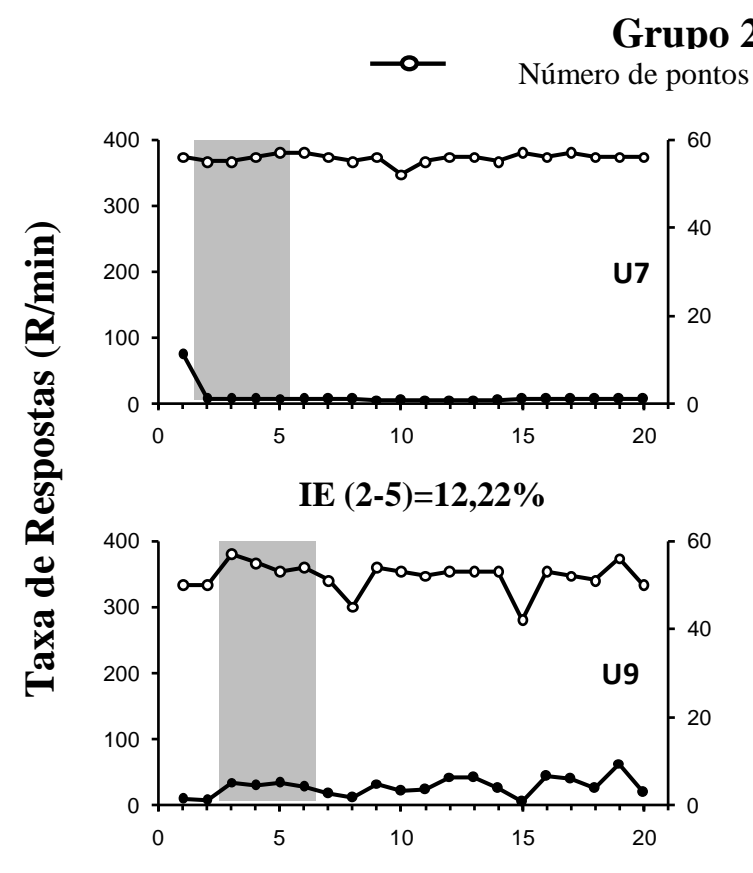

IE (3-6) $=3,83 \%$

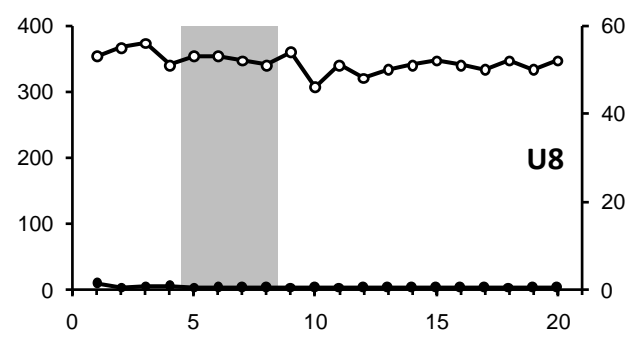

IE $(5-8)=0,57 \%$

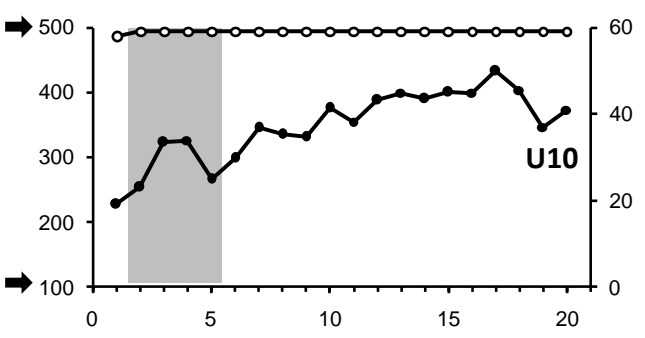

IE $(2-5)=2,90 \%$ 


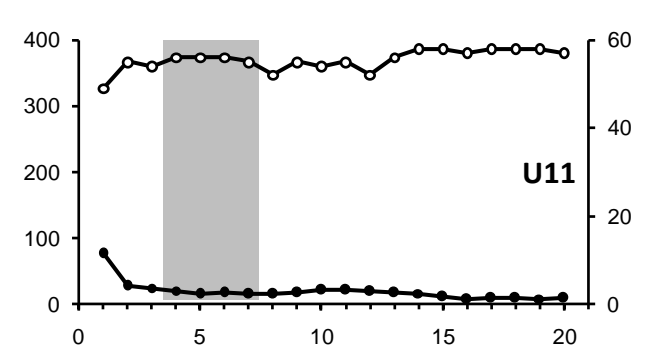

IE (4-7)=9,34\%

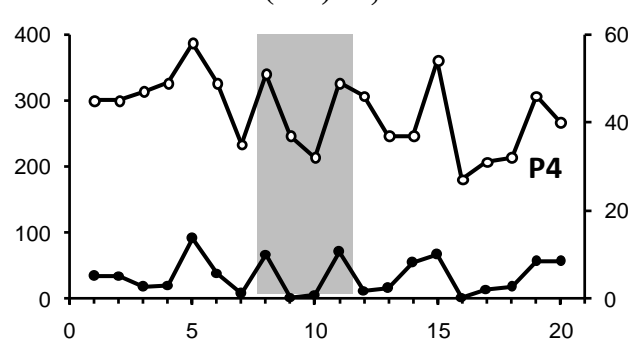

IE $(8-11)=10,40 \%$

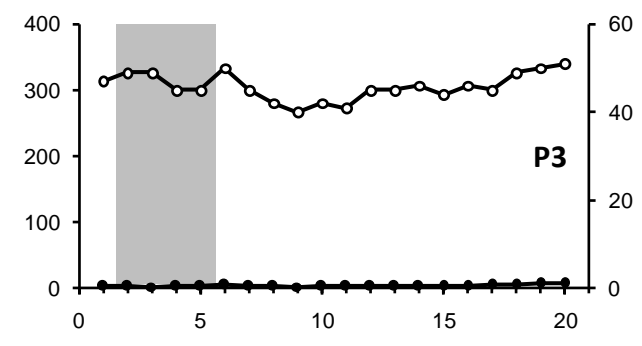

IE $(2-5)=3,02 \%$

\section{Sessões}

Figura 2. Taxa de respostas em cada sessão para os participantes do Grupo 2 - Pontos (representada pela linha com círculos preenchidos e valores no eixo y à esquerda). O número de pontos obtidos em cada sessão está representado pela linha com círculos vazios e valores no eixo y à direita. As sessões dentro dos retângulos verticais representam os blocos de sessões em que o critério de estabilidade foi atingido. O Índice de Estabilidade (IE) e o bloco de sessões em que foram alcançados (nos parênteses) estão descritos abaixo de cada gráfico. A escala do eixo y à esquerda de P12 (indicada por uma seta) é diferente das demais, inicia em $100 \mathrm{R} / \mathrm{min}$ e termina em $500 \mathrm{R} / \mathrm{min}$.

A taxa de respostas e o número de pontos obtidos também não pareceram sistematicamente relacionados ao número de sessões necessário para que o critério de estabilidade fosse alcançado. Por exemplo, U1 (Grupo 1-Dinheiro) e P3 (Grupo 2-Pontos) emitiram taxas de respostas baixas (abaixo de $13 \mathrm{R} / \mathrm{min}$ ), enquanto $\mathrm{P} 2$ (Grupo 1-Dinheiro) e U10 (Grupo 2-Pontos) emitiram taxas altas (acima de $168 \mathrm{R} / \mathrm{min}$ ) nas cinco primeiras sessões, mas esses quatro participantes atingiram o critério de estabilidade no primeiro terço do experimento. O participante U6 (Grupo 1-Dinheiro), que também emitiu baixas taxas de respostas (abaixo de $28 \mathrm{R} / \mathrm{min}$ ) ao longo das 20 sessões, atingiu o critério de estabilidade no último terço do experimento.

Em relação ao número de pontos obtidos, $\mathrm{P} 3$ ganhou menos pontos que U7 nas cinco primeiras sessões (47 e 55,8 pontos por sessão, em média, respectivamente) e ambos atingiram o critério de estabilidade no primeiro terço das 20 sessões. A análise de correlação de Pearson entre o número de sessões necessárias para que o critério de estabilidade fosse atingido, as médias das taxas de respostas e as médias de pontos obtidos nas quatro sessões de estabilidade (sessões marcadas com o retângulo cinza nas Figuras 1 e 2) não revelou correlações estatisticamente significativas para o Grupo 1 [Taxa de respostas e sessões para estabilidade ( $r=-0,483, p=0,226)$; Pontos obtidos e sessões para estabilidade $(r=0,111, p=0,794)$ ] e para o Grupo 2 [Taxa de respostas e sessões para estabilidade $(r=-0,267, p=0,563)$; Pontos obtidos e sessões para estabilidade $(r=-0,622, p=0,136)]$.

Uma diferença entre o desempenho dos participantes do Grupo 1-Dinheiro e do Grupo 2-Pontos parece ser as taxas de respostas no primeiro quarto do experimento. Quatro de oito participantes do Grupo 1Dinheiro (U2, U3, U5 e P1) responderam em taxas altas nas primeiras cinco sessões (entre 75 e 303 $\mathrm{R} / \mathrm{min}$ ) e emitiram taxas mais baixas nas últimas cinco sessões do experimento (entre 4 e $60 \mathrm{R} / \mathrm{min}$ ). Por outro lado, cinco de sete participantes do Grupo 2Pontos (U7, U8, U9, U11 e P3) responderam em taxas mais baixas (geralmente abaixo de $30 \mathrm{R} / \mathrm{min}$ ) logo nas sessões iniciais e mantiveram esse desempenho até a última sessão. Dessa forma, observa-se que, em geral, utilizar pontos trocados por dinheiro como consequência para o responder aumenta a probabilidade de 
altas taxas de respostas, principalmente quando se considera o primeiro quarto de sessões do experimento.

Os resultados do bloco de sessões em que o critério de estabilidade foi atingido pelos participantes U3, P1, P2, U10 e P4 revela uma fragilidade dos critérios de estabilidade quantitativos, como o que foi utilizado neste trabalho. Isto porque, para os participantes U3, $\mathrm{P} 1, \mathrm{P} 2$ e U10, as taxas de respostas nas três primeiras sessões do bloco de estabilidade tenderam a aumentar de uma sessão para outra e, na quarta sessão do bloco, as taxas de respostas diminuíram. O participante $\mathrm{P} 4$, por sua vez, emitiu taxas de respostas parecidas na segunda e terceira sessões, mas muito distintas da primeira e da quarta sessões do bloco em que o critério de estabilidade foi atingido. Essa fragilidade se deve à maneira como o critério de estabilidade de Schoenfeld et al. (1956) é calculado. A divisão do bloco de quatro sessões consecutivas em dois subblocos permite que as médias de cada sub-bloco sejam compostas por sessões com taxas de respostas diferentes e, no entanto, as médias desses sub-blocos podem ser parecidas.

A Figura 3 apresenta a proporção da taxa média de respostas, em blocos de quatro sessões, em relação à taxa média do bloco de quatro sessões em que o critério de estabilidade foi atingido (IE $\leq 15 \%$ - BL1). Quando o número de sessões no final da exposição ao FI foi insuficiente para compor um novo bloco de quatro sessões, essas sessões foram adicionadas ao bloco anterior, compondo blocos de, no máximo, sete sessões. As linhas cujos marcadores são quadrados cheios representam a aplicação do critério de estabilidade desde a primeira sessão, conforme os retângulos das Figuras 1 e 2. As linhas com círculos vazios representam o cálculo do critério de estabilidade quando as cinco primeiras sessões da exposição ao FI foram descartadas da análise (assim como Schoenfeld et al., 1956 e Cumming \& Schoenfeld, 1960 descartaram os sete primeiros dias de experimento com pombos como "ajustamento do pássaro" ao esquema de reforço, nesta análise optou-se por desconsiderar as cinco primeiras sessões, considerando-as como um período de adaptação às contingências de reforço). A estabilidade foi, então, novamente avaliada - a partir das sessões 6 , 7,8 e $9-$, as quatro sessões em que a estabilidade era atingida foram consideradas como o Bloco 1 e a análise seguia como anteriormente descrito. Os dados do U6 não estão representados visto que o critério de estabilidade só foi atingido na $17^{\mathrm{a}}$ sessão e, portanto, não houve sessões suficientes para compor outro bloco.

A apresentação dos dados em termos de proporção relativa de mudança (cf. Nevin, 1974, 1979), permite observar a variação da taxa média de respostas em blocos sucessivos (sempre em relação ao primeiro bloco) sem a interferência da taxa de respostas absoluta (facilitando a comparação entre participantes) e sem a variação sessão a sessão, como mostrados nas Figuras 1 e 2. A linha tracejada na Figura 3 indica uma previsão do desempenho futuro (cf. Kazdin, 1982) nos blocos de sessões após o critério de estabilidade ter sido atingido. Quanto mais as curvas se aproximam da linha tracejada, maior a manutenção da estabilidade da taxa de respostas nos blocos sucessivos de sessões. Cada 0,1 - para mais ou para menos - na curva representa uma variação de $10 \%$ na média da taxa de respostas de cada bloco em relação ao Bloco 1. 

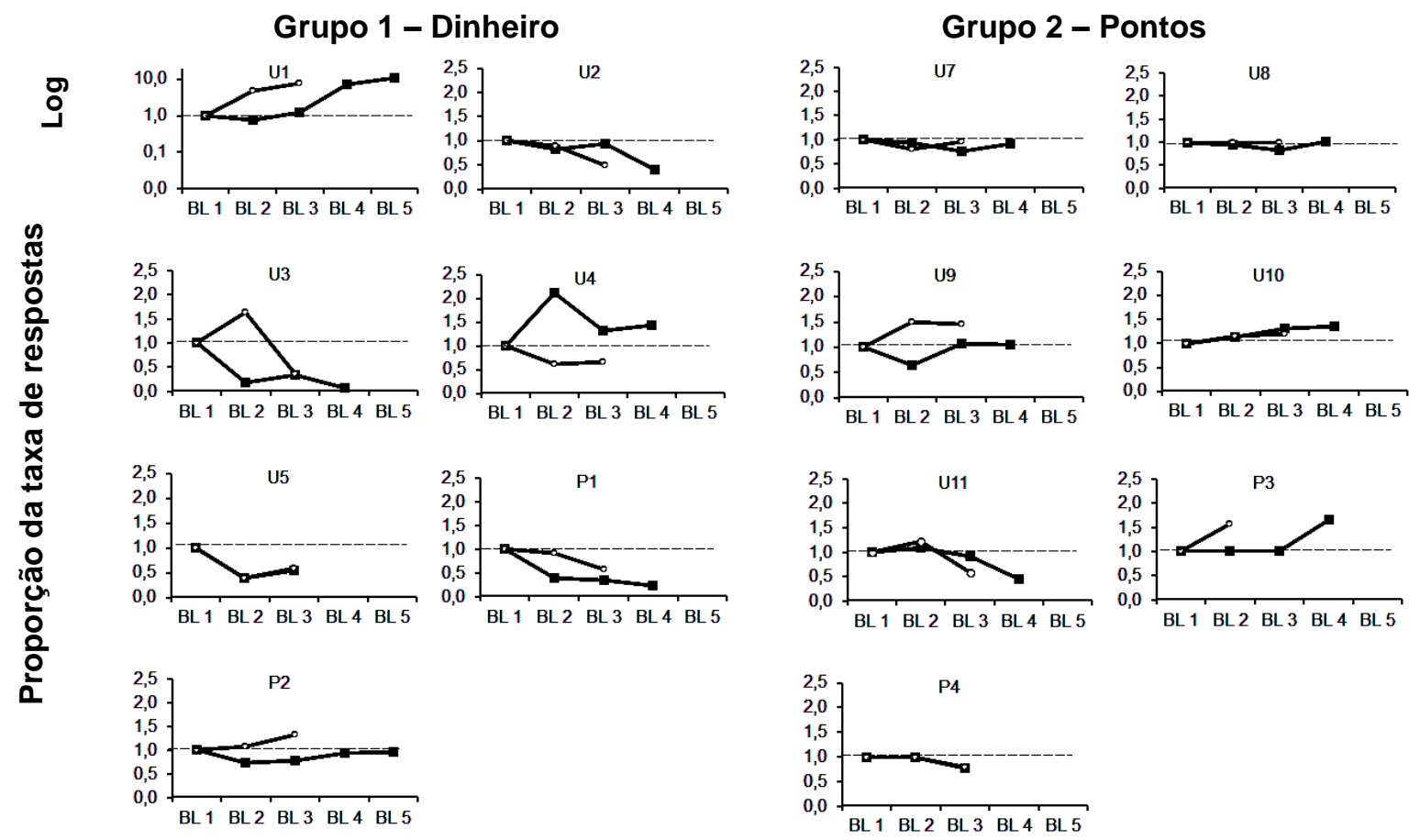

Blocos de sessão

Figura 3. Proporção da taxa média de respostas em cada bloco de quatro sessões em relação ao primeiro bloco (BL 1) no qual o índice de estabilidade foi alcançado. Nas linhas com círculos vazios, o critério de estabilidade foi calculado desconsiderando-se as cinco primeiras sessões da exposição ao FI e, nas linhas com quadrados cheios, as cinco primeiras sessões foram consideradas. Os dados do U6 (Grupo 1-Dinheiro) não estão representados, pois a estabilidade só foi atingida na $17^{\mathrm{a}}$ sessão, não havendo sessões suficientes para compor outro bloco. Apenas o eixo y do participante U1 está representado em escala logarítmica para comportar a variação da taxa média de respostas.

A taxa de respostas dos participantes do Grupo 1Dinheiro, quando comparada à do Grupo 2-Pontos, apresentou maior variação em relação ao Bloco 1 quando incluídas as cinco primeiras sessões no cálculo do critério de estabilidade (linha com quadrados preenchidos). Dentre os sete participantes do Grupo 1, quatro (U1, U3, U4 e P1) tiveram variação acima de 0,6 , dois (U2 e U5) apresentaram variação entre 0,4 e 0,6 e um (P2) manteve uma variação de até 0,3 . A variação ocorreu no sentido de redução da taxa média de respostas (em blocos sucessivos de sessões) para todos os participantes, exceto para U1 e U4 (ver também Figura 1). Para os participantes do Grupo 2Pontos, a variação foi acima de 0,6 para apenas um participante (P3); entre 0,4 e 0,6 para dois dos sete participantes (U9 e U11) e de até 0,3 para os quatro participantes restantes (U7, U8, U10 e P4).

Desconsideradas as cinco primeiras sessões para a aplicação do critério de estabilidade (linhas com círculos vazios), a variação da taxa média de respostas dos blocos posteriores ao Bloco 1 diminuiu para três dos sete participantes do Grupo 1-Dinheiro (U3, U4 e P1): a variação que estava acima de 0,6 ficou entre 0,4 e 0,6 . Para os demais participantes a variação em relação ao Bloco 1 sofreu poucas alterações em relação ao cálculo do critério de estabilidade que incluiu as cinco primeiras sessões.

Tomados em conjunto, os resultados apresentados nas Figuras 1, 2 e 3 sugerem que a consequência programada não afetou o número mínimo de sessões necessárias para que o critério estabilidade, adotado neste estudo, fosse atingido. Entretanto, a manutenção da estabilidade parece ter sido maior para os participantes do Grupo 2-Pontos do que para os participantes do Grupo 1-Dinheiro. Se as cinco primeiras sessões da exposição ao FI forem descartadas (como uma fase de adaptação), a diferença entre os grupos parece diminuir.

Em relação ao desempenho final dos participantes (Sessão 20), foi possível identificar quatro padrões 
distintos. Alguns detalhes entre o $20^{\circ}$ e o $26^{\circ}$ minuto, descritos a seguir, podem ser visualizados na Figura 4. Esse período da sessão foi escolhido porque pareceu, visualmente, o que melhor representava o padrão "tí- pico" do desempenho de cada participante na sessão final, ou seja, o desempenho que mais predominava durante toda a sessão para cada participante.

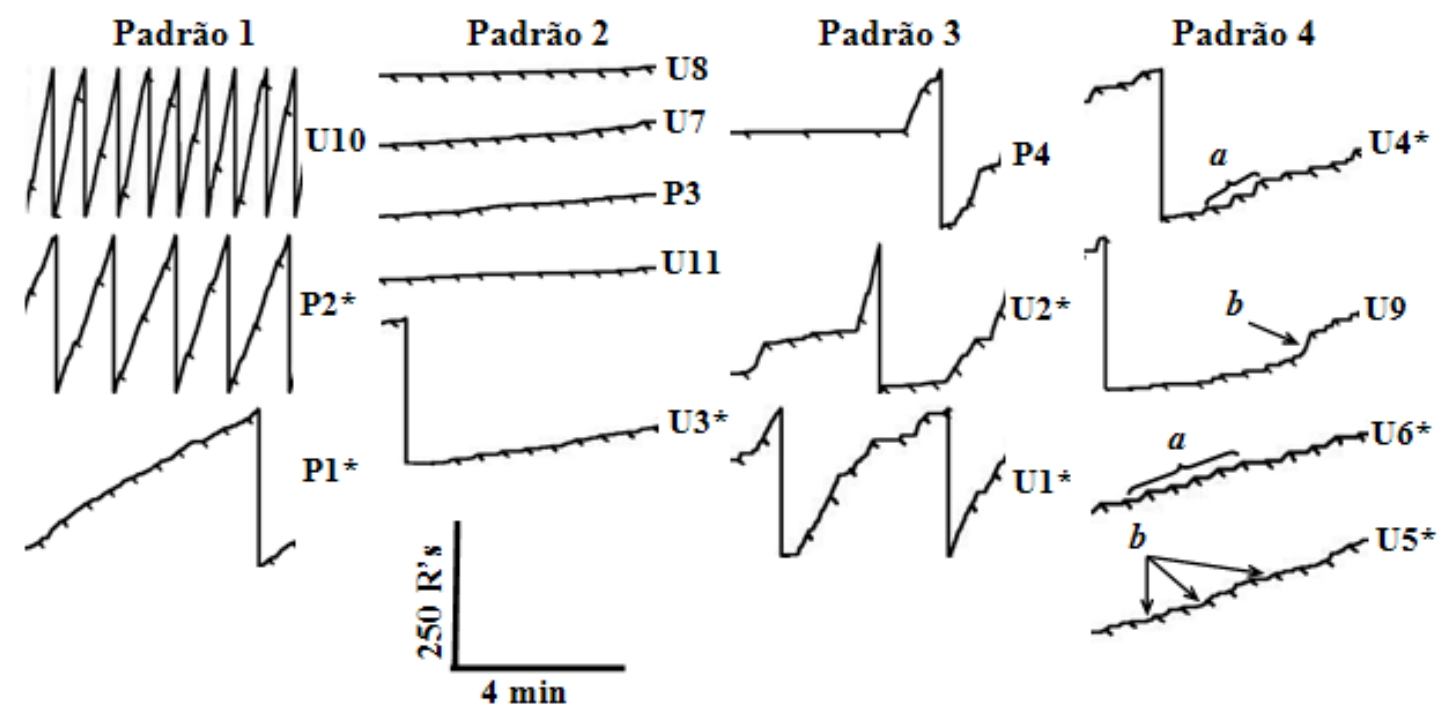

Figura 4. Frequência acumulada de respostas do $20^{\circ}$ ao $26^{\circ}$ minuto da última sessão de exposição ao FI 30 s. Os participantes estão agrupados conforme a semelhança no padrão final de respostas; aqueles com asterisco ao lado do código pertencem ao Grupo 1-Dinheiro. A curva da taxa de resposta volta a zero após ocorrência de 250 respostas. As marcas diagonais na curva da taxa de resposta indicam a liberação de pontos. A letra $a$ indica padrão de resposta em $b r e a k$-and-run e a letra $b$ indica padrão de resposta em scallop.

No Padrão 1, os participantes emitiram taxas de respostas altas e relativamente constantes, isto é, sem pausa pós-reforço (U10, P2 e P1). No Padrão 2, os participantes emitiram taxas de respostas baixas, com uma média aproximada de quatro respostas por intervalo, apesar de IRIs ocasionais com taxa relativamente mais alta (U8, U7, P3, U11 e U3). No Padrão 3 (P4, $\mathrm{U} 2$ e U1), os participantes responderam oscilando entre períodos de taxas de respostas altas e baixas. Essa oscilação entre taxas altas e baixas de respostas ocorre com predominância de períodos de alta taxa de respostas para U1; com predominância de baixas taxas de respostas para U2 e P4. No Padrão 4 (U4, U9, U6 e U5), a característica mais marcante foi o desenvolvimento de padrões de respostas em break-and-run e scallop em alguns intervalos (indicado pelas letras $a \mathrm{e}$ $b$, respectivamente, na Figura 4).

Em resumo, observa-se na Figura 4, que houve variabilidade quanto aos padrões de respostas dos participantes ao final da exposição ao FI (independentemente do grupo). No entanto, taxas de respostas mais baixas tenderam a aparecer mais frequentemente para os participantes do Grupo 2-Pontos (Padrão 2) e padrões de break-and-run e scallop foram mais frequentes para participantes do Grupo 1-Dinheiro.

\section{DISCUSSÃO}

Um dos objetivos do presente estudo foi investigar se o número de sessões necessário para que o critério de estabilidade da taxa de respostas em FI com humanos fosse atingido dependeria da consequência programada (Pontos trocados por Dinheiro vs. Pontos). Os resultados indicaram que o número de sessões necessário para que a taxa de respostas fosse considerada estável não variou muito, tanto entre participantes quanto entre grupos. No geral, a maioria dos participantes (11 de 15 participantes) atingiu o critério de estabilidade até a sétima sessão de exposição ao FI. $\mathrm{Na}$ comparação entre grupos, o número de sessões necessário para que a taxa de respostas atingisse o critério de estabilidade também não foi diferente, embora tenha havido alguma diferença individual. 
A escolha por considerar os blocos de quatro sessões, para o cálculo da estabilidade das taxas de respostas, ocorreu porque quanto menor o número de sessões mais rigoroso torna-se o critério de estabilidade (cf. Baron \& Perone, 1998; Perone, 1991), porque, como o critério calcula a porcentagem de variação em relação à média de sub-blocos de sessões, quanto maior o número de sessões, maior a probabilidade de a média esconder variações entre sessões (Perone, 1991).

Apesar de mais rigoroso, a maneira como o critério de estabilidade de Schoenfeld et al. (1956) é calculado mantém o problema das médias, isto é, a divisão do bloco de quatro sessões consecutivas em dois subblocos permite que as médias de cada sub-bloco sejam compostas por sessões com taxas de respostas diferentes e, no entanto, as médias desses sub-blocos podem ser parecidas - como pôde ser observado quando houve uma variação cíclica na taxa de respostas $(\mathrm{P} 4)$ ou quando ocorreram picos de taxas mais altas (e.g., U3, P1 e U10).

Uma medida que pode ajudar a solucionar esse problema é limitar a porcentagem de variação entre as sessões consecutivas dentro dos sub-blocos. Por exemplo, além da variação máxima entre as médias dos sub-blocos (e.g., 15\%), para que as taxas de respostas sejam consideradas estáveis, as duas sessões de cada sub-bloco também não poderiam diferir mais do que $15 \%$ entre si. Essa medida pode tornar o critério de estabilidade quantitativo de Schoenfeld et al. menos propenso a avaliar como sendo estáveis desempenhos com variação dentro dos sub-blocos.

Outro objetivo do presente estudo foi avaliar se a manutenção dos desempenhos no momento que atingiram o critério de estabilidade estaria relacionada à consequência programada. No presente estudo, a manutenção do comportamento considerado estável foi avaliada em termos da variação na taxa média de respostas dos blocos de sessões posteriores ao bloco em que o critério de estabilidade foi atingido (Figura 3). Em geral, esta variação foi menor para os participantes do Grupo 2-Pontos do que para os participantes do Grupo 1-Dinheiro (ver Figura 3 - marcadores com quadrados cheios). Portanto, é possível que a manutenção da estabilidade esteja relacionada à consequência programada. Efeitos de consequências distintas sobre o comportamento de humanos em esquemas de reforço foram descritos em outros estudos (e.g., Costa et al., 2008; Mace et al., 1997; Salgado et al., 2011; Ward, 1976; Weiner, 1972).
Entretanto, diversos autores (e.g., Baron \& Perone, 1998; Cumming \& Schoenfeld, 1960; Johnston \& Pennypacker 1993; Kazdin, 1982; Sidman, 1960) discutiram sobre a decisão experimental de considerar o desempenho estável. Uma orientação comum é "descartar" as primeiras exposições ao esquema de reforço, pois a estabilidade pode ser atingida ao acaso e não se manter no futuro. De fato, no presente estudo, a variação entre blocos de sessões, observada no desempenho de alguns participantes do Grupo 1Dinheiro (U3, U4 e P1), pareceu relacionada à redução acentuada na taxa de respostas após as cinco primeiras sessões da exposição ao FI (ver Figura 1). Como já foi observado, Schoenfeld et al. (1956) e Cumming e Schoenfeld (1960) consideraram os primeiros sete dias de experimento como "ajustamento do pássaro" ao esquema de reforço e, só então, o cálculo da estabilidade era aplicado. No presente estudo, quando foram desconsideradas da análise as cinco primeiras sessões (ver Figura 3 - marcadores com círculos vazios) e só então calculada a estabilidade (o Bloco 1 era composto pelas sessões 6 a 9), a variação entre os blocos de sessões reduziu para três de sete participantes do Grupo 1-Dinheiro (U3, U4 e P1). Nas pesquisas com humanos, questões de ordem prática tendem a levar os pesquisadores a buscar a estabilidade no menor tempo possível (Baron \& Perone, 1998). Isso pode levar os pesquisadores a não descartar as sessões iniciais, mas essa decisão pode ser problemática para a análise posterior dos resultados e até para sua replicação.

Tomados em conjunto, os resultados sobre a manutenção da estabilidade da taxa de respostas em FI parecem sugerir que ela depende da consequência programada (i.e., a estabilidade é mantida principalmente quando pontos não são trocados por nada). Entretanto, quando as sessões iniciais são descartadas a diferença engendrada pelo "tipo de reforçador" tende a diminuir e o critério de estabilidade, quando atingido, tende a ser mais preditivo do desempenho futuro. Se essa afirmação é válida para o desempenho de humanos em outros esquemas de reforço, além do FI, é uma questão que permanece em aberto.

Talvez isso ocorra porque pontos trocados por dinheiro tende a gerar taxas de respostas (inicialmente) altas. No estudo de Costa et al. (2005), os três participantes da Condição 2-Dinheiro emitiram taxas de respostas altas e constantes e na Condição 3-Pontos, dois de cinco participantes responderam em taxas altas e constantes, dois em taxas moderadas com tendência à redução e um respondeu em taxas altas inicialmente, 
mas que reduziram logo na segunda sessão. É preciso destacar que, naquele estudo, a exposição ao FI foi de apenas três sessões de 15 minutos. A exposição prolongada ao FI, realizada no presente estudo, indicou que, embora a taxa de respostas da maioria dos participantes do Grupo 1-Dinheiro fosse alta nas primeiras sessões (como em Costa et al., 2005), ela não se manteve até o final do experimento. Após, aproximadamente, cinco sessões de exposição ao FI (o que corresponde a duas horas e meia de exposição), a taxa de respostas tendeu a reduzir (embora tenha havido alguma variabilidade entre participantes nos dois grupos - ver, por exemplo, o desempenho de U1, P2, U10 e P4).

Pesquisadores adotam tempos de exposição variados, a depender do objetivo das pesquisas com humanos. Há estudos em que o tempo de exposição dependeu do número de reforços (e.g., Buskist et al., 1980, 1981); em outros, foram feitas exposições curtas, com poucas sessões de curta duração (e.g., Costa et al., 2005; Costa, Patsko \& Becker, 2007) e há estudos em que o tempo de exposição foi mais extenso, como o do presente estudo (e.g., Baron, Kaufman \& Stauber, 1969, Experimento 1; Okouchi, 2002, para quatro participantes; Weiner, 1969, Experimento 1). Os dados do presente estudo explicitam a importância de se estender a exposição em estudos com esquemas de reforço com humanos (Baron \& Perone, 1998; Bernstein, 1988), especialmente em contingência de intervalo e quando se quer garantir que o comportamento atinja estabilidade e que ela seja preditiva do comportamento futuro, se nenhuma manipulação nas contingências for realizada (cf. Kazdin, 1982; Sidman, 1960).

Mesmo com a exposição continuada à contingência de reforço, variações na taxa de respostas de humanos em FI podem ocorrer. No presente experimento, a taxa de respostas do participante U1 (Grupo 1-Dinheiro) atingiu o critério de estabilidade nas primeiras quatro sessões da exposição ao FI, mas, a partir da $13^{\mathrm{a}}$ sessão, a taxa de respostas aumentou. É possível supor, especulativamente, que alguma variável externa ao experimento tenha ganhado controle sobre o comportamento do participante ou mesmo que a diferença no total de pontos ganhos (trocados por dinheiro ao final das sessões) tenha sido responsável pela mudança na taxa de respostas, embora novas manipulações experimentais tivessem que ser realizadas para testar essas hipóteses. A média da taxa de respostas de U1 nas quatro primeiras sessões foi de $8 \mathrm{R} / \mathrm{min}$ e a média de pontos ganhos nessas sessões foi aproximadamente de
54 pontos; a média da taxa de respostas nas quatro últimas sessões do experimento foi de $86 \mathrm{R} / \mathrm{min}$ e a média de pontos ganhos foi de aproximadamente 58 pontos. A taxa de respostas de U1 estabilizou novamente (da Sessão 14 a 17) em taxas maiores do que as anteriores (média de $74 \mathrm{R} / \mathrm{min}$ ). Não é possível afirmar se o comportamento permaneceria estável com a exposição continuada ao FI.

Quanto aos padrões de respostas intrassessão (Figura 4), os resultados encontrados no presente estudo foram semelhantes aos descritos em outros estudos com humanos expostos ao FI: taxas altas e constantes, moderadas e constantes, taxas baixas com poucas respostas por intervalo e padrões de break-and-run (Baron et al., 1969; Buskist et al., 1980; Buskist et al., 1981; Costa et al., 2005; Costa et al., 2007; Okouchi, 2002; Weiner, 1969). Além desses padrões, no presente estudo, o registro cumulativo de alguns participantes mostrou padrões de scallop em alguns intervalos (Figura 4, letra $b$ ). Taxas de respostas mais baixas tenderam a aparecer mais frequentemente para os participantes do Grupo 2-Pontos (Padrão 2) e padrões break-and-run e scallop foram mais frequentes para participantes do Grupo 1-Dinheiro. Todavia, em ambos os padrões havia um participante do outro grupo com desempenho semelhante, o que sugere que a consequência programada pode aumentar a probabilidade de certos padrões, mas não os determina.

Em suma, os resultados da presente pesquisa parecem sugerir que (a) o tipo de consequência programada (Pontos vs. Pontos trocados por dinheiro) para o comportamento de humanos em FI não tem efeito sobre o número de sessões necessário para que um critério de estabilidade quantitativo da taxa de respostas seja atingido; (b) pode ser importante desconsiderar as sessões iniciais antes de aplicar o critério quando a consequência empregada são pontos trocados por dinheiro; e (c) independentemente da consequência programada (Pontos vs. Pontos trocados por Dinheiro), taxas de respostas altas e baixas podem ser selecionadas e mantidas com diversos padrões de resposta em FI com humanos, inclusive aqueles tipicamente encontrados em não-humanos como scallop e breakand-run.

\section{REFERÊNCIAS}

Baron, A., Kaufman, A., \& Stauber, K. (1969). Effects of instructions and reinforcement feedback on human operant behavior maintained by fixed-interval reinforcement. Journal of the Experimental Analysis of Behavior, 12, 701-712. 
Baron, A., \& Perone, M. (1998). Experimental design and analysis in the laboratory study of human operant behavior. In K. A. Lattal \& M. Perone (Eds.), Handbook of research methods in human operant behavior (pp. 45-91). New York: Plenum Press.

Bentall, R. P., Lowe, C. F., \& Beasty, A. (1985). The role of verbal behavior in human learning: II. Developmental differences. Journal of the Experimental Analysis of Behavior, 43, 165-181.

Bernstein, D. (1988). Laboratory lore and research practices in the experimental analysis of human behavior: Designing session logistic - How long, how often, how many? The Behavior Analyst, 11, 51-58.

Buskist, W. F., Bennett, R. H., \& Miller, H. L. (1981). Effects of instrucional constraints on human fixed-interval performance. Journal of the Experimental Analysis of Behavior, 35, 217-225.

Buskist, W. F., Miller, H. L., \& Bennett, R. H. (1980). Fixed-interval performance in humans: Sensitivity to temporal parameters when food is the reinforcer. The Psychological Record, 30, 111-121.

Costa, C. E., \& Banaco, R. A. (2002). ProgRef v3: Sistema computadorizado para a coleta de dados sobre programas de reforço com humanos - Recursos básicos. Revista Brasileira de Terapia Comportamental e Cognitiva, 4, 171-172.

Costa, C. E., Banaco, R., \& Becker, R. M. (2005). Desempenho em FI com humanos: Efeito do tipo de reforçador. Temas em Psicologia da SBP, 13, 14-29.

Costa, C. E., Banaco, R., Longarezi, D. M., Martins, E. V., Maciel, E. M., \& Sudo, C. H. (2008). O tipo de reforçador como variável moduladora dos efeitos da história em humanos. Psicologia: Teoria e Pesquisa, 24, 251-262.

Costa, C. E. \& Cançado, C. R. X. (2012). Stability Check: A program for calculating the stability of behavior. Mexican Journal of Behavior Analysis, 38, 61-71.

Costa, C. E., Patsko, C. H., \& Becker, R. M. (2007). Desempenho em FI com humanos: Efeito da interação da resposta de consumação e do tipo de instrução. Interação em Psicologia, 11, 175-186.

Cumming, W. W., \& Schoenfeld, W. N. (1958). Behavior under extended exposure to a high-value fixed interval reinforcement schedule. Journal of the Experimental Analysis of Behavior, 1, 245-263.

Cumming, W. W., \& Schoenfeld, W. N. (1960). Behavior stability under extended exposure to a time correlated reinforcement contingency. Journal of the Experimental Analysis of Behavior, $3,71-82$.

Ferster, C. B., \& Skinner, B. F. (1957). Schedules of reinforcement. New York, NY: Appleton-Century Crofts.

Freeman, T. J., \& Lattal, K. A. (1992). Stimulus control of behavioral history. Journal of the Experimental Analysis of Behavior, 57, 5-15.
Galizio, M. (1979). Contingency-shaped and ruled-governed behavior: Instructional control of human loss avoidance. Journal of the Experimental Analysis of Behavior, 31, 53-70.

Johnston, J. M., \& Pennypacker, H. S. (1993). Strategies and tatics of behavioral research. Hillsdale, NJ: Erlbaum.

Kazdin, A. E. (1982). Single-case research designs: Methods for clinical and applied settings. New York, NY: Oxford University Press.

Lattal, K. A. (1970). Relative frequency of reinforcement and rate of punished behavior. Journal of the Experimental Analysis of Behavior, 13, 319-324.

Mace, F. C., Mauro, B. C., Boyajian, A. E., \& Eckert, T. L. (1997). Effects of reinforcer quality on behavioral momentum: Coordinated applied and basic research. Journal of Applied Behavior Analysis, 30, 1-20.

Matos, M. A. (1990). Controle experimental e controle estatístico: A filosofia do caso único na pesquisa comportamental. Ciência e Cultura, 42, 585-592.

Matthews, B. A., Shimoff, E., Catania, A. C., \& Sagvolden, T. (1977). Uninstructed human responding: Sensitivity to ratio and interval contingencies. Journal of the Experimental Analysis of Behavior, 27, 453-467.

Nevin, J. A. (1974). Response strength in multiple schedules. Journal of the Experimental Analysis of Behavior, 21, 389-408.

Nevin, J. A. (1979). Reinforcement schedules and response strength. In M. D. Zeiler \& P. Harzem (Eds.), Reinforcement and the organization of behavior (pp. 117-158). Chichester, England: Wiley.

Okouchi, H. (2002). Individual differences in human fixedinterval performance. The Psychological Record, 12, 173-186.

Perone, M. (1991). Experimental design in the analysis of freeoperant behavior. In I. H. Iversen \& K. A. Lattal (Eds.), Experimental analysis of behavior - Part 1 (pp. 135-171). Amsterdam, NL: Elsevier.

Salgado, R. C., Cançado, C. R. X., \& Costa, C. E. (2011). Efeitos do reforçador empregado em história de reforço recente e remota. Psicologia: Teoria e Pesquisa, 27, 119-130.

Schoenfeld, W. N., Cumming, W. W., \& Hearst, E. (1956). On the classification of reinforcement schedules. Proceedings of the National Academy of Sciences of the United States of America, 42, 563-570.

Sidman, M. (1960). Tactics of scientific research: Evaluating experimental data in psychology. New York, NY: Basic Books.

Ward, J. (1976). Variation of reinforcement in performance of a motor skill. Perceptual an Motor Skills, 43, 149-150.

Weiner, H. (1964). Conditioning history and human fixed-interval performance. Journal of the Experimental Analysis of Behavior, 7, 383-385.

Weiner, H. (1969). Controlling human fixed-interval performance. Journal of the Experimental Analysis of Behavior, 12, 349-373. 
Weiner, H. (1972). Human fixed-ratio responding as a function of the type of reinforcer (money vs. points) and the presence or absence of a noncontingent monetary wage. The Psychological

Recebido em 25/08/2012 Record, 21, 497-500.

Notas:

1 O artigo foi derivado da dissertação de mestrado do Programa de Mestrado em Análise do Comportamento da Universidade Estadual de Londrina do primeiro autor sob a supervisão do terceiro autor.

2 O break-and-run consiste de uma pausa, geralmente após o reforço, e uma transição abrupta na taxa de respostas de uma frequência próxima de zero para uma frequência constante que é mantida até o final do intervalo (Cumming \& Schoenfeld, 1958). Outro padrão característico do FI, o scallop, consiste de uma pausa logo após o reforço, seguido por uma taxa de respostas positivamente acelerada até a liberação do próximo reforço (Ferster \& Skinner, 1957).

3 O projeto foi aprovado pelo Comitê de Ética em Pesquisa Envolvendo Seres Humanos da Universidade Estadual de Londrina sob o número 283/07. 